\title{
Radical-Radical interaction through a saturated link:
}

\section{Methylene-bis-6-oxoverdazyl}

\author{
David J. R. Brook* and Gordon T. Yee \\ Department of Chemistry, Santa Clara University, Santa Clara CA 95053. \\ Department of Chemistry, Virginia Polytechnic and State University, Blacksburg, VA 24061
}

\section{Supporting Information}

\section{Contents}

General Experimental S1

Malondialdehyde Bisulfite Addition Compound …………..................................... S2

Computational Results for Structure 6 (triplet) ...................................................... S3

References

General. 2,4-Diisopropyl-carbonohydrazide bis-hydrochloride was synthesized as previously described. ${ }^{1}$ NMR spectra were recorded on a $300 \mathrm{MHz}$ FTNMR spectrometer and referenced to residual solvent proton signals. IR spectra were recorded on thin films on $\mathrm{NaCl}$ using an FTIR spectrometer. Gas chromatograms and mass spectra were recorded on a GCMS fitted with a 5\% phenyl methyl siloxane $30.0 \mathrm{~m} \times 250 \mu \mathrm{m} \times 0.25 \mu \mathrm{m}$ column. The carrier gas was He flowing at $1.5 \mathrm{~mL} / \mathrm{min}$. Samples were injected as solutions in dichloromethane, ether or toluene. The GC oven was held at $70^{\circ} \mathrm{C}$ for $2 \mathrm{~min}$, ramped to $300^{\circ} \mathrm{C}$ at $20^{\circ} \mathrm{C} / \mathrm{min}$. and held at $300^{\circ} \mathrm{C}$ for $10 \mathrm{~min}$. The detector was a quadrupole MS with $70 \mathrm{eV}$ electron impact ionization. UV-visible spectra were recorded on a diode array spectrophotometer using a combined tungsten filament/deuterium 
lamp light source. Electrochemical measurements used a computer controlled potentiostat with platinum disk working electrode, platinum wire counter electrode and $\mathrm{Ag}^{+} / \mathrm{Ag} / \mathrm{CH}_{3} \mathrm{CN}$ reference. The supporting electrolyte was $0.1 \mathrm{M} \mathrm{Bu}_{4} \mathrm{~N}^{+} \mathrm{ClO}_{4}^{-}$in acetonitrile. The ferrocene/ferricinium couple was used as an internal reference. ESR spectra were recorded on an X band spectrometer equipped with a frequency counter. The magnetic field was calibrated with an external $\mathrm{MnO}$ in $\mathrm{CaO}$ sample. Samples were degassed by three freeze-(77K)-pump-(0.1 Torr)-thaw cycles before measurement. The magnetic properties of compound $\mathbf{5}$ were determined by SQUID magnetometry. Approximately $15 \mathrm{mg}$ was placed between cotton plugs inside a gelatin capsule. Magnetization as a function of temperature was obtained by zero field cooling and measuring on warming from $5 \mathrm{~K}$ in $5000 \mathrm{G}$. Diamagnetic corrections for the cotton and capsule were applied. The diamagnetic contribution of the sample was estimated using Pascal's constants. Semiempirical calculations were performed using Spartan PC-Pro or MOPAC 7 running on a pentium 4 desktop or Macintosh Powerbook G4 laptop computer respectively.

\section{Malondialdehyde sodium bisulfite addition compound.}

This was synthesized from 1,1,3,3-tetramethoxypropane as described by Saslaw and Waravdekar. ${ }^{2}$ The product mixture contains both meso and $d l$ isomers, the predominant $d l$ isomer (80\% of the sample by integration) with ${ }^{1} \mathrm{H}$ NMR $\left(\mathrm{D}_{2} \mathrm{O}\right) 2.27$ (2H, AA' part of AA'XX' system, $\left.\mathrm{J}_{\mathrm{AB}}=5.5 \mathrm{~Hz}, \mathrm{~J}_{\mathrm{AB}}=7.4 \mathrm{~Hz}\right), 4.59\left(2 \mathrm{H}, \mathrm{XX}\right.$ ' part of $\mathrm{AA}^{\prime} \mathrm{XX}$ ' system $) ;{ }^{13} \mathrm{C} \mathrm{NMR}\left(\mathrm{D}_{2} \mathrm{O}\right) 31.2,77.9$. The meso isomer (20\% by integration) had ${ }^{1} \mathrm{H}$ NMR $\left(\mathrm{D}_{2} \mathrm{O}\right) 2.09$ (A part of $\mathrm{AMX}_{2}$ system, $\left.\mathrm{J}_{\mathrm{AM}}=14.7 \mathrm{~Hz}, \mathrm{~J}_{\mathrm{AX}}=7.9 \mathrm{~Hz}\right) 2.58\left(\mathrm{M}\right.$ part of $\mathrm{AMX}_{2}$ system, $\left.\mathrm{J}_{\mathrm{MX}}=5.5 \mathrm{~Hz}\right), 4.67\left(\mathrm{X}\right.$ part of $\mathrm{AMX}_{2}$ system $){ }^{13} \mathrm{C}$ NMR $\left(\mathrm{D}_{2} \mathrm{O}\right) 32.3,79.1$ 
Computational Results for Structure 6 (triplet)

CARTESIAN COORDINATES

\begin{tabular}{|c|c|c|c|c|}
\hline NO. & ATOM & $X$ & $\mathrm{Y}$ & Z \\
\hline 1 & $\mathrm{C}$ & .0000 & .0000 & .0000 \\
\hline 2 & $\mathrm{~N}$ & 1.4830 & .0000 & .0000 \\
\hline 3 & $\mathrm{~N}$ & 2.1100 & 1.2211 & .0000 \\
\hline 4 & $\mathrm{C}$ & 3.4691 & 1.2090 & -.0384 \\
\hline 5 & $\mathrm{C}$ & 4.1787 & 2.3774 & .0458 \\
\hline 6 & $\mathrm{C}$ & 3.6876 & 3.7454 & .1880 \\
\hline 7 & $\mathrm{~N}$ & 2.3698 & 4.0525 & -.1453 \\
\hline 8 & $\mathrm{~N}$ & 1.8951 & 5.3309 & .3570 \\
\hline 9 & $\mathrm{C}$ & 2.8032 & 6.3073 & .6824 \\
\hline 10 & $\mathrm{~N}$ & 4.1133 & 5.9314 & .7511 \\
\hline 11 & $\mathrm{~N}$ & 4.5438 & 4.5968 & .5835 \\
\hline 12 & $\mathrm{C}$ & 5.0733 & 6.8531 & 1.3755 \\
\hline 13 & $\mathrm{C}$ & 6.4344 & 6.7768 & .6811 \\
\hline 14 & $\mathrm{H}$ & 7.0925 & 7.5391 & 1.0848 \\
\hline 15 & $\mathrm{H}$ & 6.8835 & 5.8073 & .8391 \\
\hline 16 & $\mathrm{H}$ & 6.3170 & 6.9521 & -.3814 \\
\hline 17 & $\mathrm{C}$ & 5.1663 & 6.5651 & 2.8805 \\
\hline 18 & $\mathrm{H}$ & 5.8122 & 7.2950 & 3.3528 \\
\hline 19 & $\mathrm{H}$ & 4.1835 & 6.6360 & 3.3352 \\
\hline 20 & $\mathrm{H}$ & 5.5683 & 5.5730 & 3.0481 \\
\hline 21 & $\mathrm{H}$ & 4.6458 & 7.8280 & 1.2269 \\
\hline 22 & 0 & 2.4475 & 7.4544 & .9097 \\
\hline 23 & $\mathrm{C}$ & .6097 & 5.7914 & -.2078 \\
\hline 24 & $\mathrm{C}$ & .7585 & 6.1759 & -1.6835 \\
\hline 25 & $\mathrm{H}$ & 1.4960 & 6.9634 & -1.7898 \\
\hline 26 & $\mathrm{H}$ & 1.0648 & 5.3195 & -2.2703 \\
\hline 27 & $\mathrm{H}$ & -.1904 & 6.5468 & -2.0545 \\
\hline 28 & $\mathrm{C}$ & -.4670 & 4.7316 & .0221 \\
\hline 29 & $\mathrm{H}$ & -1.4438 & 5.1356 & -.2155 \\
\hline 30 & $\mathrm{H}$ & -.3033 & 3.8716 & -.6155 \\
\hline 31 & $\mathrm{H}$ & -.4686 & 4.4262 & 1.0627 \\
\hline 32 & $\mathrm{H}$ & .3742 & 6.6719 & .3678 \\
\hline 33 & $\mathrm{H}$ & 1.7204 & 3.3216 & .0794 \\
\hline 34 & $\mathrm{H}$ & 5.2405 & 2.2955 & .0608 \\
\hline 35 & $\mathrm{~N}$ & 4.2159 & .0611 & -.1280 \\
\hline 36 & $\mathrm{~N}$ & 3.4984 & -1.1155 & -.1937 \\
\hline 37 & $\mathrm{C}$ & 2.1368 & -1.2019 & -.1147 \\
\hline 38 & 0 & 1.5388 & -2.2637 & -.1443 \\
\hline 39 & $\mathrm{C}$ & 4.2898 & -2.3652 & -.2612 \\
\hline 40 & $\mathrm{C}$ & 4.9848 & -2.6065 & 1.0800 \\
\hline 41 & $\mathrm{H}$ & 5.5004 & -3.5596 & 1.0491 \\
\hline 42 & $\mathrm{H}$ & 5.7032 & -1.8220 & $1.276^{\circ}$ \\
\hline
\end{tabular}




$\begin{array}{rrrrr}43 & \mathrm{H} & 4.2573 & -2.6369 & 1.8848 \\ 44 & \mathrm{C} & 5.2758 & -2.3002 & -1.4261 \\ 45 & \mathrm{H} & 4.7504 & -2.0840 & -2.3504 \\ 46 & \mathrm{H} & 6.0176 & -1.5351 & -1.2486 \\ 47 & \mathrm{H} & 5.7742 & -3.2581 & -1.5304 \\ 48 & \mathrm{H} & 3.5598 & -3.1371 & -.4378 \\ 49 & \mathrm{C} & -.5375 & .8355 & 1.1611 \\ 50 & \mathrm{H} & -.1168 & .4878 & 2.0982 \\ 51 & \mathrm{H} & -.2953 & 1.8781 & 1.0234 \\ 52 & \mathrm{H} & -1.6169 & .7383 & 1.2145 \\ 53 & \mathrm{C} & -.4953 & .4759 & -1.3662 \\ 54 & \mathrm{H} & -.1959 & 1.5021 & -1.5338 \\ 55 & \mathrm{H} & -.0794 & -.1493 & -2.1493 \\ 56 & \mathrm{H} & -1.5760 & .4095 & -1.4132 \\ 57 & \mathrm{H} & -.2663 & -1.0329 & .1438\end{array}$

AM1 CALCULATION

MOPAC VERSION 7.00

Tue Mar 14 07:04:23 2006

FINAL HEAT OF FORMATION =

$166.41928 \mathrm{KCAL}$

$\begin{array}{llc}\text { TOTAL ENERGY } & = & -4878.12679 \mathrm{EV} \\ \text { ELECTRONIC ENERGY } & = & -40983.66384 \mathrm{EV} \\ \text { CORE-CORE REPULSION } & = & 36105.53705 \mathrm{EV} \\ & & \\ \text { IONIZATION POTENTIAL } & = & 4.98378 \\ \text { NO. OF FILLED LEVELS } & = & 2 \\ \text { AND NO. OF OPEN LEVELS } & = & \end{array}$

\section{References}

1) Paré, E. C.; Brook, D. J. R.; Brieger, A.; Badik, M.; Schinke, M., Org. Biomol. Chem. 2005, 3, 4258-4261.

2) Saslaw, L. D.; Waravdekar, V. S., J. Org. Chem. 1957, 22, 843-844. 